\title{
The inhomogeneous ISM toward PKS 1830-211 SW: A detailed view of molecular gas at a look-back time of $7.5 \mathrm{Gyr}^{\star}$
}

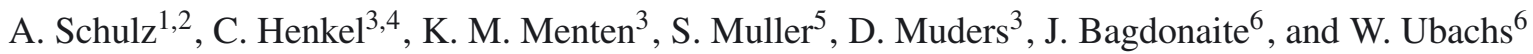 \\ 1 Argelander-Institut für Astronomie, Universität Bonn, Auf dem Hügel 71, 53121 Bonn, Germany \\ e-mail: chenkel@mpifr-bonn.mpg.de \\ 2 Institut für Physik und ihre Didaktik, Universität zu Köln, Gronewaldstr. 2, 50931 Köln, Germany \\ ${ }^{3}$ Max-Planck-Institut für Radioastronomie, Auf dem Hügel 69, 53121 Bonn, Germany \\ 4 Astron. Dept., King Abdulaziz University, PO Box 80203, 21589 Jeddah, Saudi Arabia \\ 5 Dept. of Earth and Space Sciences, Chalmers University, Onsala Space Observatory, 43992 Onsala, Sweden \\ ${ }^{6}$ Dept. of Physics and Astronomy, VU University Amsterdam, De Boelelaan 1081, 1081 HV Amsterdam, The Netherlands \\ Received 28 September 2014 / Accepted 4 December 2014
}

\section{ABSTRACT}

\begin{abstract}
Based on measurements with the Effelsberg 100-m telescope, a multiline study of molecular species is presented toward the southwestern source of the gravitational lens system PKS 1830-211, which is by far the best known target for studying molecular gas in absorption at intermediate redshift. Determining line parameters and optical depths and performing large velocity gradient radiative transfer calculations, the aims of this study are (1) to evaluate physical parameters of the absorbing foreground gas at $z \sim 0.89$, in particular its homogeneity; and (2) to monitor the spectroscopic time variability caused by fluctuations in the $z \sim 2.5$ background continuum source. We find, that the gas is quite inhomogeneous with $n\left(\mathrm{H}_{2}\right) \sim 2 \times 10^{3} \mathrm{~cm}^{-3}$ for most molecular species but with higher values for $\mathrm{H}_{2} \mathrm{CO}$ and lower ones for SO. Measuring the CS $J=1 \leftarrow 0$ transition during a time interval of more than a decade, from 2001 to 2012, the peak absorption depth of the line remains approximately constant, while the line shape undergoes notable variations. Covering the time between 1996 and 2013, $\mathrm{CS}, \mathrm{HCO}^{+}$, and $\mathrm{CH}_{3} \mathrm{OH}$ data indicate maximum integrated optical depths in 2001 and 2011/2012. This is compatible with a $\sim 10 \mathrm{yr}$ periodicity, which, however, needs confirmation by substantially longer time monitoring. Comparing molecular abundances with those of different types of Galactic and nearby extragalactic clouds we find that the observed cloud complex does not correspond to one particular type but to a variety of cloud types with more diffuse and denser components as can be expected for an observed region with a transverse linear scale of several parsec and a likely greater depth along the line of sight. A tentative detection of Galactic absorption in the $\mathrm{c}-\mathrm{C}_{3} \mathrm{H}_{2} 1_{10}-1_{01}$ line at $18.343 \mathrm{GHz}$ is also reported.
\end{abstract}

Key words. galaxies: abundances - galaxies: ISM - galaxies: individual: PKS 1830-211 SW - gravitational lensing: strong radio lines: galaxies - radio lines: ISM

\section{Introduction}

To study molecular cloud properties in far distant galaxies, low spatial resolution becomes an increasingly important problem since observed spectra provide averages over large areas. Furthermore, beam filling factors are small causing low flux densities and, therefore, low signal-to-noise ratios. A suitable way to overcome these problems is to use molecular lines in absorption in front of compact background continuum sources such as radio quasars whose small angular sizes determine the effective beam size of the observation and whose continuum flux densities determine the sensitivity of the measurements. The availability of such background-foreground pairs and the required strength of the background continuum limit the application of this method to a very small number of objects. The so far brightest and most distant such pair is PKS 1830-211 (Wiklind \& Combes 1996a), a gravitational lens with truly unique properties. The background source is likely a blazar at $z=2.507$ (Lidman et al. 1999; De Rosa et al. 2005). The foreground lens is a spiral galaxy observed almost face-on at redshift $z \sim 0.89$ (Courbin et al. 2002; Winn et al. 2002). It splits the continuum radiation of the blazar into three main image components.

\footnotetext{
* The spectra (FITS files) are only available at the CDS via anonymous ftp to cdsarc.u-strasbg. fr (130.79.128.5) or via http://cdsarc.u-strasbg.fr/viz-bin/qcat?]/A+A/574/A108
}

These consist of a stronger northeastern "hotspot", a weaker southwestern "hotspot", and an Einstein ring that is prominent only at low frequencies $(<10 \mathrm{GHz}$; Jauncey et al. 1991). The southwestern source is located behind a spiral arm of the lensing galaxy, at a distance of about $2 \mathrm{kpc}$ from its center (Muller et al. 2006), and shows by far the strongest molecular absorption lines among all known sources of its class (for the others, see Wiklind \& Combes 1994, 1995, 1996b; and Kanekar et al. 2005).

The exceptional situation concerning continuum flux and line strength encountered in PKS 1830-211 has been extensively explored, leading to a large number of detected molecular lines and species (e.g., Henkel et al. 2009; Muller et al. 2011, 2014a,b). Therefore it provides detailed insight into the properties of the interstellar medium (ISM) of a galaxy at an intermediate cosmological distance. Adopting a standard $\Lambda$-cosmology with $H_{0}=67 \mathrm{~km} \mathrm{~s}^{-1} \mathrm{Mpc}^{-1}, \Omega_{\mathrm{m}}=0.315$, and $\Omega_{\Lambda}=0.685$ (Planck Collaboration XVI 2014), $z=0.88582$ corresponds to a lookback time of about $7.5 \mathrm{Gyr}$, more than half the present age of the Universe.

It is the aim of this article to examine physical properties of the molecular gas using molecular lines characterizing various physical conditions. This includes a first evaluation of the homogeneity of the absorbing molecular gas column and CS $J=1 \leftarrow 0$ spectra covering more than a decade to study variability caused by the background continuum source. 
Table 1. Observational parameters.

\begin{tabular}{ccccc}
\hline \hline $\begin{array}{c}\nu \\
(\mathrm{GHz})\end{array}$ & $\begin{array}{c}\lambda \\
(\mathrm{cm})\end{array}$ & $\begin{array}{c}\theta_{\mathrm{b}}{ }^{a} \\
\left({ }^{\prime \prime}\right)\end{array}$ & $\begin{array}{c}T_{\text {sys }}{ }^{b} \\
(\mathrm{~K})\end{array}$ & $\eta_{\mathrm{A}}{ }^{c}$ \\
\hline $13.5-18.7$ & 1.9 & $60-45$ & 40 & $0.39-0.32$ \\
$18.0-26.0$ & 1.3 & $45-35$ & 70 & $0.33-0.26$ \\
$27.0-36.7$ & 1.0 & $32-23$ & 75 & $\sim 0.30$ \\
\hline
\end{tabular}

Notes. ${ }^{(a)}$ Full width to half power (FWHP) beam width in arcsec. ${ }^{(b)}$ System temperatures in units of antenna temperature $\left(T_{\mathrm{A}}^{*}\right)$. (c) Aperture efficiency.

\section{Observations}

The observations were carried out in a position-switching mode with the primary focus $\lambda=1.9,1.3$, and $1.0 \mathrm{~cm}$ receivers of the 100 -m telescope at Effelsberg/Germany ${ }^{1}$, mostly within the short period between August and December 2001. On- and offsource integration times were two minutes per scan, yielding full cycles of four to five minutes duration. For full width to half power (FWHP) beam widths, system temperatures, and aperture efficiencies, see Table 1. During 2001-2006, the backend was an 8192 channel autocorrelator, which was split into eight segments, covering selected frequency ranges within a total bandwidth of $500 \mathrm{MHz}$. Each of the eight segments had a bandwidth of $40 \mathrm{MHz}$ with 512 channels, yielding channel spacings of $0.7-$ $1.7 \mathrm{~km} \mathrm{~s}^{-1}$. At $\lambda \sim 1.3 \mathrm{~cm}$, but not at 1.9 and $1.0 \mathrm{~cm}$, a dual channel receiver was used to sample orthogonal linear polarizations. Therefore, in this case at least two of the eight segments were centered at the same frequency to maximize sensitivity by observing the two polarization components simultaneously. In 2006, a fast Fourier transform spectrometer (FFTS) was employed with a bandwidth of $100 \mathrm{MHz}$ and 16384 channels for each polarization channel. For the more recent CS data from April 2011 to April 2012 another FFTS was used, this time with a bandwidth of $2 \mathrm{GHz}$ and 32768 channels per polarization. At $26 \mathrm{GHz}$, the corresponding FFTS channel spacings are 0.07 and $0.7 \mathrm{~km} \mathrm{~s}^{-1}$, respectively. For recent (2012/3) methanol measurements, the 32768 channel FFTS has also been used, but in this case with a bandwidth of $100 \mathrm{MHz}$. Details of these recent methanol observations can be found in Bagdonaite et al. (2013a,b).

As already mentioned (Sect. 1), PKS 1830-211 is with 5 Jy near $\lambda=1 \mathrm{~cm}$ one of the strongest continuum sources in the radio sky. Therefore, focus and pointing could be checked toward the source itself. The latter was found to be accurate to about $5^{\prime \prime}$. The continuum was also used to calibrate the spectral lines to obtain line-to-continuum flux density ratios, which could be determined with an accuracy of up to $\pm 7 \%$ depending on the strength of the respective line (see also Sect. 4). Data reduction was obtained using standard procedures of CLASS and GREG, two tools of the GILDAS ${ }^{2}$ software package. During the data reduction, only linear baselines were subtracted from the original spectra.

\section{Results}

The separation between the northeastern and southwestern hotspots of PKS 1830-211 (see Sect. 1) is about 1 arcsec.

\footnotetext{
1 The 100-m telescope at Effelsberg is operated by the Max-PlanckInstitut für Radioastronomie (MPIfR) on behalf of the Max-PlanckGesellschaft (MPG).

2 Grenoble Image and Line Data Analysis Software, see http://www . iram. fr/IRAMFR/GILDAS
}

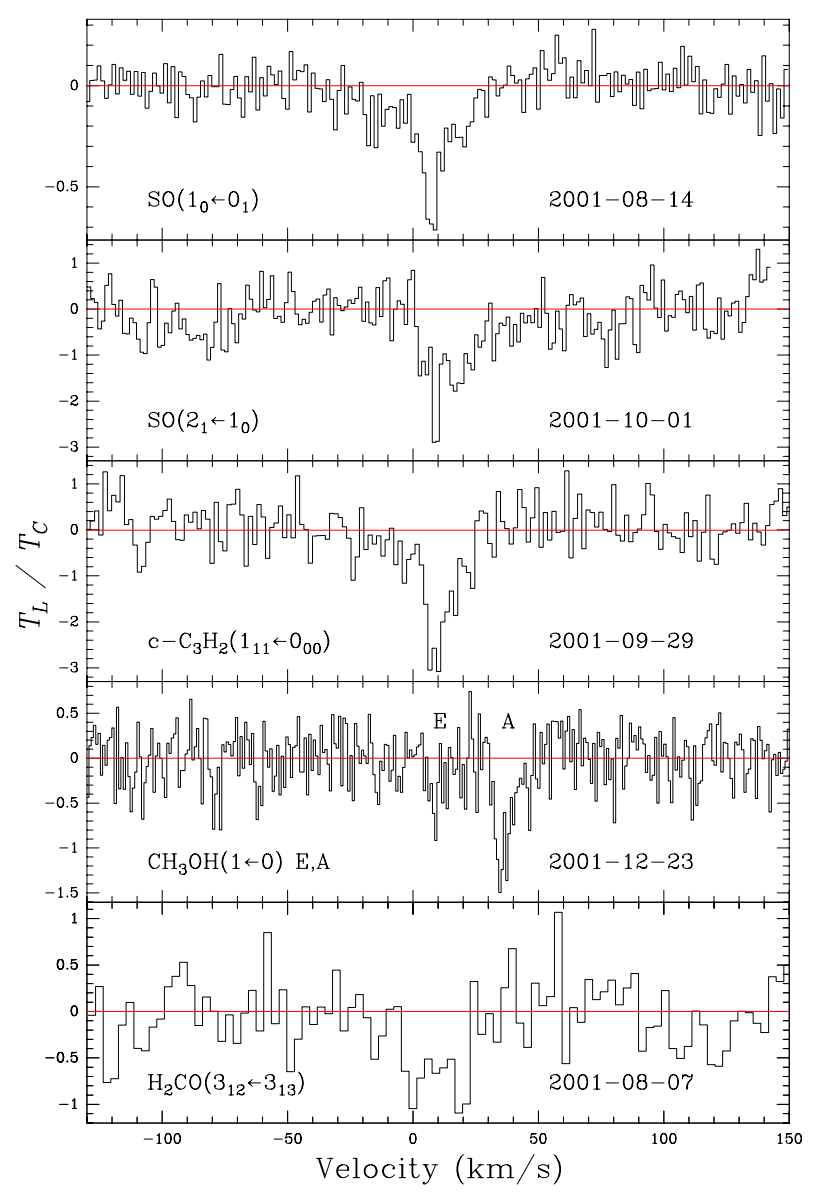

Fig. 1. Six absorption lines detected toward PKS 1830-211, displayed in five panels with a local standard of rest (LSR) velocity scale relative to $z=0.88582\left(V_{\mathrm{LSR}}=V_{\mathrm{HEL}}+12.4 \mathrm{~km} \mathrm{~s}^{-1}\right.$; ordinate: absorption depth in units of percent of the observed continuum flux density, $T_{\mathrm{C}}$, accounting for the entire source). The spectra were taken between August and December 2001. The velocity scale of the fourth panel from the top is based on the E-transition of methanol $\left(\mathrm{CH}_{3} \mathrm{OH}\right)$. The scale for the A-transition is offset by $27.5 \mathrm{~km} \mathrm{~s}^{-1}$. The cyclic- $\mathrm{C}_{3} \mathrm{H}_{2}$ line of the central panel belongs to the para species (see Table 2). Channel spacings are $1.4,1.4,1.7,0.9$ and $3.0 \mathrm{~km} \mathrm{~s}^{-1}$ from top to bottom.

Therefore, they are clearly within our much larger beam sizes (Table 1). Figure 1 shows the line profiles measured at Effelsberg between August and December 2001, with the ordinate displaying absorption in units of percent of the total observed continuum flux density. The abscissa depicts local standard of rest (LSR) velocity relative to $z=0.88582$. The detected absorption lines all originate in the southwestern hotspot. We have obtained five spectra (one containing two lines) of the molecules $\mathrm{SO}$, para-c- $\mathrm{C}_{3} \mathrm{H}_{2}, \mathrm{CH}_{3} \mathrm{OH}$, and ortho- $\mathrm{H}_{2} \mathrm{CO}$. While, in the meantime, these molecules have also been detected in other studies (e.g., Muller et al. 2011), these are the earliest detections except for $\mathrm{H}_{2} \mathrm{CO}$ and $\mathrm{C}_{3} \mathrm{H}_{2}$ (Menten et al. 1999). The spectra do not show absorption line components at an LSR velocity of about $-135 \mathrm{~km} \mathrm{~s}^{-1}$, which might appear in front of the NE hotspot (Wiklind \& Combes 1998; Muller et al. 2006). Figure 2 depicts our tentative detection of Galactic $\mathrm{C}_{3} \mathrm{H}_{2}$, which would be the first detection of Galactic molecular gas absorption toward the line of sight to PKS 1830-211.

Apparent optical depths are determined using

$\tau_{\text {app }}=-\ln \left(1-\frac{\left|T_{\mathrm{L}}\right|}{T_{\mathrm{C}}}\right)$ 
Table 2. Line parameters and derived optical depths.

\begin{tabular}{|c|c|c|c|c|c|c|c|c|c|}
\hline Transition & \multicolumn{2}{|c|}{$(\mathrm{GHz})$} & \multicolumn{2}{|c|}{$\begin{array}{c}V \\
\left(\mathrm{~km} \mathrm{~s}^{-1}\right) \\
\end{array}$} & $f_{\mathrm{c}}$ & $\tau$ & $\begin{array}{c}\tau \\
f_{\mathrm{c}}=.38\end{array}$ & $\Delta \tau$ & Remarks \\
\hline \multicolumn{10}{|c|}{ Our detections } \\
\hline $\mathrm{H}_{2} \mathrm{CO} 3_{12} \leftarrow 3_{13}(\mathrm{o})$ & 15.36456 & 28.97480 & $8.9 \pm 2.7$ & $27.1 \pm 5.3$ & .153 & .054 & .021 & $30 \%$ & 070801 \\
\hline \multirow[t]{2}{*}{ SO $1_{0} \leftarrow 0_{1}$} & 15.90902 & 30.00154 & $7.5 \pm 0.4$ & $5.4 \pm 1.2$ & .156 & .031 & .013 & $10 \%$ & 140801,1 \\
\hline & & & $5.8 \pm 2.4$ & $37.2 \pm 5.8$ & .156 & .016 & .006 & $25 \%$ & 140801,1 \\
\hline $\mathrm{c}-\mathrm{C}_{3} \mathrm{H}_{2} 1_{10} \leftarrow 1_{01}(\mathrm{o})$ & 18.34314 & 18.34314 & $4.6 \pm 0.6$ & $5.1 \pm 1.5$ & 1.00 & .007 & - & $\gtrsim 30 \%$ & 151201,2 \\
\hline $\mathrm{CH}_{3} \mathrm{OH} 1_{0} \leftarrow 0_{0}\left(\mathrm{~A}^{+}\right)$ & 25.65063 & 48.37247 & $8.7 \pm 0.5$ & $8.3 \pm 1.4$ & .196 & .065 & .033 & $20 \%$ & 231201,3 \\
\hline $\mathrm{CH}_{3} \mathrm{OH} 1_{0} \leftarrow 0_{0}(\mathrm{E})$ & 25.65297 & 48.37689 & $8.7 \pm 1.3$ & $5.1 \pm 4.9$ & .196 & .020 & .010 & $100 \%$ & 231201,3 \\
\hline $\mathrm{c}-\mathrm{C}_{3} \mathrm{H}_{2} 1_{11} \leftarrow 0_{00}(\mathrm{p})$ & 27.49012 & 51.84141 & $10.2 \pm 0.7$ & $15.4 \pm 2.3$ & .198 & .097 & .050 & $20 \%$ & 290901 \\
\hline SO $2_{1} \leftarrow 1_{0}$ & 33.37105 & 62.93179 & $8.6 \pm 1.1$ & $20.9 \pm 2.5$ & .227 & .063 & .038 & $20 \%$ & 011001 \\
\hline \multicolumn{10}{|c|}{ Our nondetections } \\
\hline $\mathrm{HC}_{5} \mathrm{~N} 13 \leftarrow 12$ & 18.35508 & 34.61439 & - & - & .17 & $<.003$ & $<.001$ & $7 \%$ & 151201,4 \\
\hline $\operatorname{SiS} 2 \leftarrow 1$ & 19.25403 & 36.30963 & - & - & .17 & $<.002$ & $<.001$ & $7 \%$ & 151201,4 \\
\hline $\mathrm{H} 56 \alpha$ & 19.33709 & 36.46627 & - & - & .17 & $<.018$ & $<.008$ & $7 \%$ & 151201,4 \\
\hline $\mathrm{H} 70 \beta$ & 19.49376 & 36.76172 & - & - & .17 & $<.018$ & $<.008$ & $7 \%$ & 151201,4 \\
\hline $\mathrm{HNCO} 2_{02} \leftarrow 1_{01}$ & 23.31249 & 43.96300 & - & - & .19 & $<.004$ & $<.002$ & $7 \%$ & 301201,4 \\
\hline $\mathrm{HC}_{5} \mathrm{~N} 20 \leftarrow 19$ & 28.23830 & 53.25235 & - & - & .21 & $<.005$ & $<.003$ & $7 \%$ & 031001,4 \\
\hline $\mathrm{SO}_{2} 2_{11} \leftarrow 2_{02}$ & 28.38493 & 53.52887 & - & - & .22 & $<.006$ & $<.004$ & $7 \%$ & 031001,4 \\
\hline $\mathrm{SO}_{3} \leftarrow 3_{3}$ & 35.01657 & 66.03494 & - & - & .23 & $<.009$ & $<.006$ & $7 \%$ & 021001,4 \\
\hline \multicolumn{10}{|c|}{ Menten et al. (1999) } \\
\hline $\mathrm{H}_{2} \mathrm{CO} 2_{11} \leftarrow 22_{12}(\mathrm{o})$ & 07.68285 & 14.48848 & $\sim 9$ & $\sim 20$ & .20 & .050 & .026 & $15 \%$ & 5 \\
\hline $\mathrm{c}-\mathrm{C}_{3} \mathrm{H}_{2} 2_{12} \leftarrow 1_{01}(\mathrm{o})$ & 45.25294 & 85.33891 & $\sim 9$ & $\sim 20$ & .35 & .210 & .190 & $15 \%$ & 5,7 \\
\hline \multicolumn{10}{|c|}{ Muller et al. (2011) } \\
\hline $\mathrm{c}-\mathrm{C}_{3} \mathrm{H}_{2} 3_{31} \leftarrow 3_{22}(\mathrm{p})$ & 31.58196 & 59.55789 & - & - & .22 & .005 & .003 & $20 \%$ & 6 \\
\hline $\mathrm{CH}_{3} \mathrm{OH} 1_{0} \leftarrow 2_{-1}(\mathrm{E})$ & 32.09821 & 60.53146 & $7.1 \pm 0.5$ & $21.2 \pm 1.2$ & .22 & .033 & .019 & $10 \%$ & 6 \\
\hline $\mathrm{SO} 2_{1} \leftarrow 1_{0}$ & 33.37105 & 62.93180 & $11.4 \pm 0.3$ & $19.7 \pm 0.7$ & .23 & .050 & .030 & $10 \%$ & 6 \\
\hline $\mathrm{c}-\mathrm{C}_{3} \mathrm{H}_{2} 2_{02} \leftarrow 1_{11}(\mathrm{p})$ & 43.53202 & 82.09356 & - & - & .26 & .084 & .057 & $10 \%$ & 6 \\
\hline $\mathrm{c}-\mathrm{C}_{3} \mathrm{H}_{2} 3_{12} \leftarrow 3_{03}(\mathrm{o})$ & 43.99476 & 82.96621 & $11.5 \pm 0.1$ & $18.7 \pm 0.2$ & .26 & .031 & .022 & $10 \%$ & 6 \\
\hline $\mathrm{c}-\mathrm{C}_{3} \mathrm{H}_{2} 2_{12} \leftarrow 1_{01}(\mathrm{o})$ & 45.25294 & 85.33891 & - & - & .26 & .285 & .189 & $10 \%$ & 6,7 \\
\hline $\mathrm{SO} 2_{2} \leftarrow 1_{1}$ & 45.65332 & 86.09395 & - & - & .27 & .004 & .003 & $35 \%$ & 6 \\
\hline
\end{tabular}

Notes. "Our detections" and "Our nondetections" were all obtained between August and December 2001. Col. (1): molecular line, (o) = ortho, (p) = para; Col. (2): redshifted frequency, adopting $z=0.88582$; Col. (3): approximate rest frequency; Cols. (4) and (5): local standard of rest velocities and full width to half power (FWHP) line widths, in case of SO $1_{0} \leftarrow 0_{1}$ for both the broad and the narrow component; Cols. (6) and (7): continuum source-covering factor (see also Sect. 4.1 and Henkel et al. 2009) and corresponding peak optical depth(s); Col. (8): peak optical depths for a continuum source-covering factor of 0.38; Col. (9): estimated $1 \sigma$ uncertainties in percent of the optical depth. Remarks (last column): the 6-digit numbers provide the epoch of our observations with day, month, and year from left to right. 1: possibly two line components. 2: Galactic line, tentative detection. A continuum source-covering factor of unity has been adopted. 3: E- and A-transitions seen in the same spectrum (Fig. 1). The intensities are consistent with a line strength ratio of 1:3.8. 4: upper $1 \sigma$ limits of $\tau$; spectra were smoothed to $\sim 10 \mathrm{~km} \mathrm{~s}^{-1}$ channels. 5: extrapolated continuum flux densities: $7.1 \mathrm{Jy}(14 \mathrm{GHz})$ and $3.5 \mathrm{Jy}$ (45 GHz). 6: calculated from Table 10 of Muller et al. (2011). 7: $\tau$ values of Menten et al. (1999) and Muller et al. (2011) agree well.

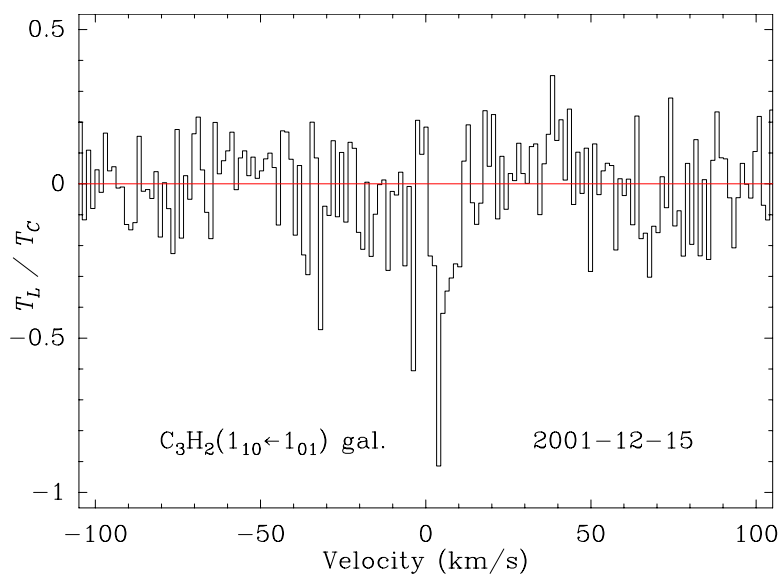

Fig. 2. Tentative detection of Galactic $\mathrm{c}-\mathrm{C}_{3} \mathrm{H}_{2}$ (cyclic variant, orthospecies), with a channel spacing of $1.3 \mathrm{~km} \mathrm{~s}^{-1}$. The spectrum was taken on December 15, 2001. The ordinate denotes absorption in units of percent of the entire observed continuum flux density, $T_{\mathrm{C}}$, of PKS 1830-211. The abscissa is local standard of rest velocity for redshift zero. (e.g., Henkel et al. 2009), with $T_{\mathrm{L}}$ and $T_{\mathrm{C}}$ denoting line and continuum temperature and assuming (realistically) that the excitation temperature $T_{\mathrm{ex}}$ is negligible relative to $T_{\mathrm{C}}$ (see also Sect. 4.4). Line parameters and apparent optical depths are given in Table 2. The table also contains upper limits to the optical depths of six molecular transitions and two recombination lines, which were also obtained between August and December 2001, and additional data taken from the literature. The noise level of the data with detected absorption does not allow us to distinguish between several velocity components as it has been done, for example, by Henkel et al. (2009) when analyzing their CS $J=1 \leftarrow 0$ profile.

Within the limits of noise, all the main line components obtained in 2001 show similar velocities (see also Henkel et al. 2008, 2009 for additional lines at $\lambda \sim 1-2 \mathrm{~cm}$ ). The local standard of rest (LSR) velocities of the peaks of the absorption (LSR velocities will be used throughout the paper), are close to $\sim 9-10 \mathrm{~km} \mathrm{~s}^{-1}$. Figure 3 displays CS $J=1 \leftarrow 0$ spectra from the southwestern component taken over a time interval of more than a decade, from 2001 to 2012 . Table 3 summarizes the corresponding line parameters. 

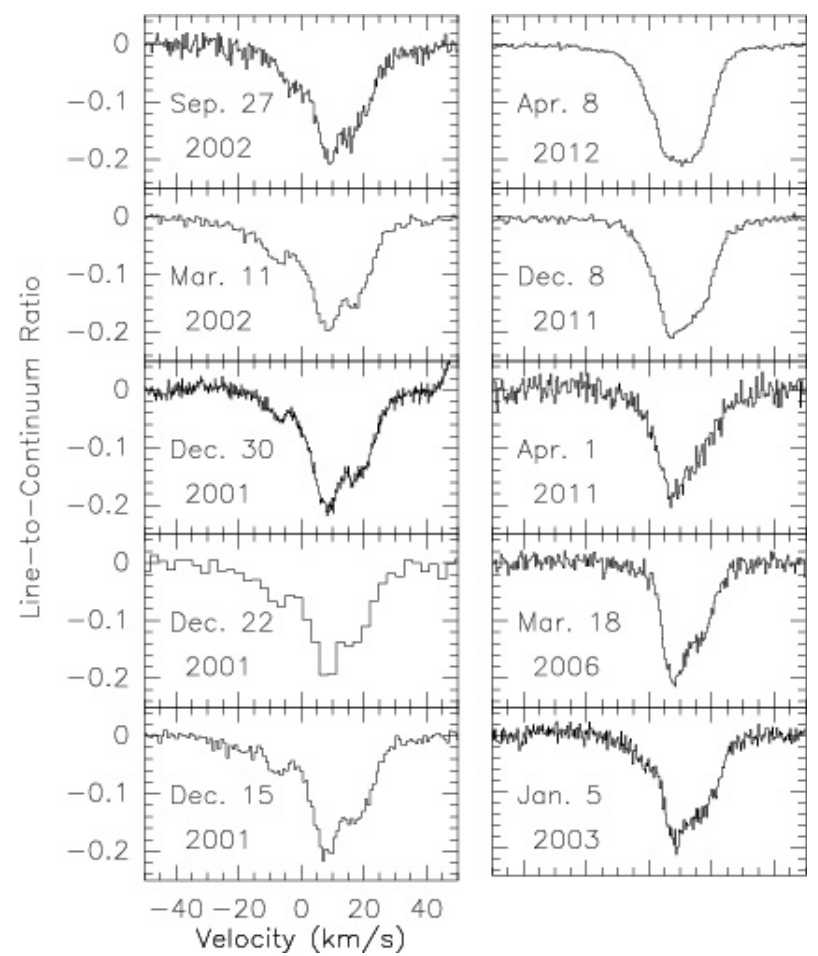

Fig. 3. Monitoring observations of the CS $(1 \leftarrow 0)$ line near $26 \mathrm{GHz}$, covering more than a decade (from lower left to upper right). Channel spacings are (from the earliest to the latest epoch) 0.90, 2.7, 0.22, 0.90, 0.45, $0.34,0.45,0.42,0.70$, and $0.70 \mathrm{~km} \mathrm{~s}^{-1}$. The ordinate denotes absorption in units of the entire continuum flux density of PKS 1830-211. The abscissa is local standard of rest velocity for redshift $z=0.88582$.

Table 3. CS $J=1 \leftarrow 0$ one component Gaussian fit parameters.

\begin{tabular}{cccc}
\hline \hline Epoch & $\int\left(T_{\mathrm{L}} / T_{\mathrm{c}}\right) \mathrm{d} V$ & $\begin{array}{c}V_{\mathrm{LSR}} \\
\left(\mathrm{km} \mathrm{s}^{-1}\right)\end{array}$ & $\Delta V$ \\
\hline Dec. 15, 2001 & $-4.43 \pm 0.07$ & $10.6 \pm 0.2$ & $23.5 \pm 0.5$ \\
Dec. 22, 2001 & $-4.42 \pm 0.11$ & $09.8 \pm 0.3$ & $24.9 \pm 0.8$ \\
Dec. 30, 2001 & $-4.38 \pm 0.05$ & $11.3 \pm 0.1$ & $22.1 \pm 0.3$ \\
Mar. 11, 2002 & $-4.76 \pm 0.05$ & $09.6 \pm 0.1$ & $26.4 \pm 0.3$ \\
Sep. 27, 2002 & $-4.42 \pm 0.06$ & $11.0 \pm 0.1$ & $23.4 \pm 0.4$ \\
Jan. 05, 2003 & $-3.57 \pm 0.04$ & $11.5 \pm 0.1$ & $19.1 \pm 0.2$ \\
Mar. 18, 2006 & $-3.13 \pm 0.04$ & $10.9 \pm 0.1$ & $15.5 \pm 0.2$ \\
Apr. 01. 2011 & $-3.32 \pm 0.04$ & $09.9 \pm 0.1$ & $18.3 \pm 0.3$ \\
Dec. 08, 2011 & $-4.28 \pm 0.03$ & $10.3 \pm 0.1$ & $19.4 \pm 0.1$ \\
Apr. 08, 2012 & $-4.60 \pm 0.02$ & $10.1 \pm 0.1$ & $20.0 \pm 0.1$ \\
\hline
\end{tabular}

Notes. Adopted redshifted frequency: $v=25.978596 \mathrm{GHz}$. The absorption peak is slightly (typically $1.0-2.5 \mathrm{~km} \mathrm{~s}^{-1}$ ) blueshifted with respect to the velocities given in Col. 3, caused by the asymmetry in the overall lines (Fig. 3). For integrated optical depths, see Fig. 4.

\section{Analysis}

\subsection{Source-covering factor and time variability}

To determine the true optical depths of our spectra from the southwestern source, an important problem to be discussed is the background source coverage factor $f_{\mathrm{c}}$. Muller et al. (2006) find their $\mathrm{HCO}^{+}$feature at $94 \mathrm{GHz}$ being saturated at a depth of $38 \%$ relative to the total continuum level implying $f_{\mathrm{c}}=0.38$ at this frequency (see also Martí-Vidal et al. 2013; and Muller et al. 2014b). Trying to account for a larger extent of the background continuum at lower frequencies, Henkel et al. (2009) have proposed a frequency dependence of this factor, $f_{\mathrm{c}}=$ $0.2 \times\left(v_{\text {observed }} / 26 \mathrm{GHz}\right)^{0.5}$ for $v_{\text {observed }} \lesssim 100 \mathrm{GHz}$, matching what has been determined by Muller et al. (2006) at the high frequency end. Therefore, we present calculated $\tau_{\text {peak }}$ values using both, the constant coverage factor of 0.38 and the frequencydependent factor in our Table 2. Nevertheless, this affects our analysis only marginally since below we use ratios of optical depths that lead to results that are quite independent of coupling values because frequencies are not too discrepant.

Another problem discussed in the literature are concerns about the variability of the source (e.g., Muller \& Guélin 2008). Henkel et al. (2009) find that the depth of the absorption features is correlated well with the continuum flux. Therefore variations in the ratio of line intensity to continuum flux remain negligible for the lines displayed in Fig. 1. These data (but not the CS $J=1 \leftarrow 0$ spectra shown in Fig. 3) were collected within a short period during 2001 (see also Sect. 2 and Table 2), which is confined to epochs within the observing period covered by Henkel et al. (2009).

A further important point is whether this argumentation also holds when using data taken over a longer time span. This seems to be the case because two lines relevant for this study were observed at two largely different epochs: (1) the ortho $c-\mathrm{C}_{3} \mathrm{H}_{2}$ $2_{12} \leftarrow 1_{01}$ transition, reported by Muller et al. (2011), was also observed by Menten et al. (1999). The agreement in peak optical depths is excellent (see Table 2). (2) The SO $2_{1} \leftarrow 1_{0}$ line observed by us in 2001 was also observed by Muller et al. (2011), again yielding consistent results within the error limits. We have to note, however, that this agreement may be fortuitous and would not necessarily hold, if we compared data from other epochs.

To better illustrate this effect, Fig. 3 shows a series of CS $(1 \leftarrow 0)$ lines measured between Dec. 2001 and Apr. 2012. The depth of the absorption remains constant to within the calibration uncertainties (see Sect. 2), while the line shape is slowly varying. The component at about $-10 \mathrm{~km} \mathrm{~s}^{-1}$ fades in 2002 . The one at about $+18 \mathrm{~km} \mathrm{~s}^{-1}$ disappears between 2006 and 2011 . In April 2012, the profile becomes almost Gaussian, suggesting that the velocity range with a significant optical depth (greater than unity) has increased. Comparing these data with the observations by Muller et al. (2008) of the even more saturated $\mathrm{HCO}^{+} J=2 \leftarrow 1$ line (their data are presented on a heliocentric velocity scale) also shows a roughly constant absorption depth (implying that $f_{\mathrm{c}}$ remains approximately constant in time, see also Muller et al. 2006; Henkel et al. 2009), the fading of the $-10 \mathrm{~km} \mathrm{~s}^{-1}$ component, and a trend to a more Gaussian profile near the end of their monitoring period (May 2007).

To visualize the changes, Fig. 4 displays the integrated optical depths of two main molecular tracers, $\mathrm{HCO}^{+} J=2 \leftarrow 1$ and CS $1 \leftarrow 0$, the latter amplified by a factor of four, to match the other data. Since $\mathrm{HCO}^{+}$shows higher optical depths than CS (see Muller \& Guélin 2008; Henkel et al. 2009; Muller et al. 2014b), the blue wings of the $\mathrm{HCO}^{+}$profiles have been plotted, which show significant variablity, indicating only moderate saturation as for CS. The observed trend in integrated line opacity variations shows excellent agreement. To expand the monitored time interval further, we have also included recent methanol $\left(\mathrm{CH}_{3} \mathrm{OH}\right)$ data from Effelsberg (see also Sect. 2), which were published by Bagdonaite et al. (2013a,b) and which show a significant decrease in integrated opacities from a peak value reached at the end of 2011 or early 2012 . Combining these data, we see peaks in 2000-2002 and 2011-2012 and lower values at other times, when considering the time interval between 1995 and 2013. This suggests a period of variability on the order 


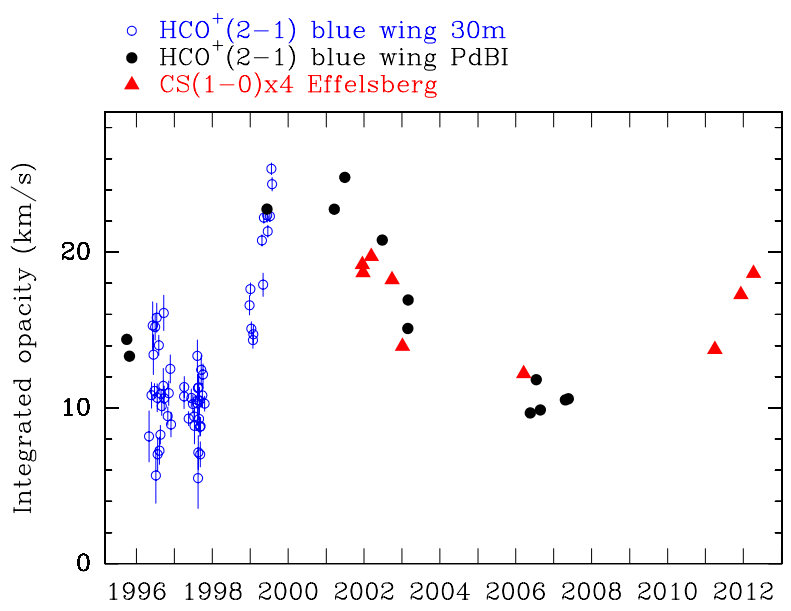

Fig. 4. Integrated opacities for $f_{\mathrm{c}}=0.38$ of the blue wing of the $\mathrm{HCO}^{+} J=2 \leftarrow 1$ lines $\left(-37.6 \mathrm{~km} \mathrm{~s}^{-1}<V_{\mathrm{LSR}}<-2.6 \mathrm{~km} \mathrm{~s}^{-1}\right.$; $\left.V_{\mathrm{LSR}}=V_{\mathrm{HEL}}+12.4 \mathrm{~km} \mathrm{~s}^{-1} ; z=0.88582\right)$ and of CS $J=1 \leftarrow 0$ (see also Fig. 3 and Table 3). The $\mathrm{HCO}^{+}$spectra were taken from IRAM Pico Veleta $(30-\mathrm{m})$ and Plateau de Bure (PdBI) data published by Muller \& Guélin (2008). Their calibration uncertainties are $15 \%$ and $1 \%$, respectively. For the Effelsberg data, see Sect. 2.

of $10 \mathrm{yr}$. Of course, having obtained data from less than two such full periods, this is speculative. Nevertheless, longer term monitoring might yield periodicities that could be related to activity of the background blazar. Accompanied by systematic measurements revealing the spatial fine structure of the line absorption and background continuum, this could probe the activity at the base of a blazar's jet (see, e.g., Nair et al. 2005), also testing the plasmon ejection model proposed by Martí-Vidal et al. (2013).

We thus agree with Muller et al. (2011) that the line shape experiences gradual changes which result in variations of velocityintegrated optical depths. Peak optical depths appear to be less variable than integrated optical depths, but we note that both the $\mathrm{HCO}^{+} 2 \leftarrow 1$ line (Muller et al. 2008) and, at the very center, our CS $1 \leftarrow 0$ line show signs of saturation, while below optically thin transitions will be discussed. Nevertheless, in view of occasionally measured broad features with questionable significance, we prefer to focus in the following on peak line intensities and the corresponding optical depths. The measured absorption is better defined by the depth of the (main) feature than by integrated opacities with sometimes poorly defined line widths. The most recent data of Muller et al. (2013) are not considered here because Muller et al. (2013) report significant variations with respect to their 2009/10-spectra, in good agreement with our findings from $\mathrm{CS} 1 \leftarrow 0$ and $\mathrm{CH}_{3} \mathrm{OH}$ (see Figs. 3-5).

\subsection{Radiative transfer calculations}

Among our observed spectra from 2001, only SO is detected in two different transitions. However, to perform radiative transfer modeling for all of our observations, we need at least two different lines for each molecule. We therefore take line data from two other investigations from which we can derive line center optical depths, from Muller et al. (2011) for $\mathrm{HNCO}, \mathrm{CH}_{3} \mathrm{OH}-\mathrm{E}$, para and ortho $\mathrm{c}-\mathrm{C}_{3} \mathrm{H}_{2}$ (their Table 10) and Menten et al. (1999) for ortho $\mathrm{c}-\mathrm{C}_{3} \mathrm{H}_{2}$ and $\mathrm{H}_{2} \mathrm{CO}$. As already mentioned in Sect. 4.1 (see also Table 2), these data are compatible, because peak opacities are similar. SO $2_{1} \leftarrow 1_{0}$ opacities from Muller et al. (2011) agree with our data ten years earlier and the same is the case for c- $\mathrm{C}_{3} \mathrm{H}_{2} 2_{12} \leftarrow 1_{01}$ opacities, when comparing Menten et al. (1999) and Muller et al. (2011).

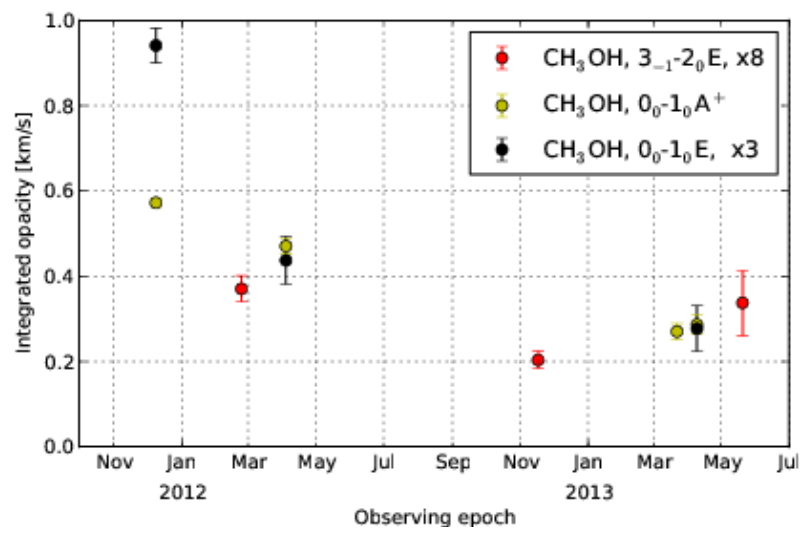

Fig. 5. Integrated opacities of three optically thin methanol $\left(\mathrm{CH}_{3} \mathrm{OH}\right)$ transitions adopting a continuum source covering factor $f_{\mathrm{c}}=0.38$. The data were obtained at Effelsberg (for details, see Bagdonaite et al. 2013a,b) and are shown to demonstrate the decline in opacities during $2012 / 3$ in a qualitative way. The $1_{0} \leftarrow 0_{0}$ E and the $2_{0} \leftarrow 3_{-1}$ E opacities were multiplied by (arbitrary) factors of 3 and 8, to bring values of all lines to roughly the same y-axis scale. Calibration uncertainties are of order $10 \%$ or smaller.

To reproduce line ratios of the observed molecules, we apply the standard large velocity gradient (LVG) model described by Henkel et al. (2009) assuming a spherically symmetric cloud and constant kinetic temperature and density throughout the emitting region. On one hand, all the molecular lines discussed below absorb less than three percent of the background continuum flux. On the other hand, the continuum source-covering factor lies above 0.15 (Muller \& Guélin 2008; Henkel et al. 2009; Muller et al. 2014b). We can therefore be certain (as already suggested in Sect. 4.1) that all the lines are optically thin. This implies that our results do not depend on cloud morphology. Therefore, our assumption of spherical cloud geometry will not affect the results.

In contrast to the work of Muller et al. (2013), we do not assume single values of gas density and temperature common for all molecules in our analysis, which they themselves regard as the major limitation of their excitation analysis. We have carried out our model calculations for a wide variety of densities and temperatures (see Figs. 6-10) that examine also those obtained by Henkel et al. (2008, 2009) and Muller et al. (2011).

All data used for the model calculations are contained in Table 2. The basic question is whether the results are consistent with the density and temperature parameters of previous studies, i.e. $T_{\text {kin }}=50$ or $80 \mathrm{~K}$ and $n\left(\mathrm{H}_{2}\right)=1000-2000 \mathrm{~cm}^{-3}$ (Henkel et al. 2008, 2009; Muller et al. 2011, 2013), the former involving the classical $T_{\text {kin }}$ tracers $\mathrm{NH}_{3}$ and $\mathrm{CH}_{3} \mathrm{CN}$. Because the common degeneracy between high $T_{\text {kin }} /$ low $n\left(\mathrm{H}_{2}\right)$ and vice versa affects most molecular line pairs, including our tracers, there is a wide parameter space for each pair of lines. Here we thus intend to find out whether the above-mentioned $T_{\text {kin }} / n\left(\mathrm{H}_{2}\right)$ combination can reproduce our data in all cases. The results discussed below are valid for both sets of continuum coverage factors (Sect. 4.1 and Table 2), which slightly affect our results only in cases of significantly diverging frequencies of the lines involved.

$c-C_{3} H_{2}$ : (p): the observed $2_{02} \leftarrow 1_{11}$ to $1_{11} \leftarrow 0_{00}$ ratio of para-cyclopropenylidene, $0.87 \pm 0.19\left(f_{\mathrm{c}}=0.198\right)$ or $1.14 \pm 0.25\left(f_{\mathrm{c}}=0.38\right)$, is - also for the former value within $2 \sigma-$ compatible with $T_{\text {kin }}=50-80 \mathrm{~K}$ and $n\left(\mathrm{H}_{2}\right)=1000-2000 \mathrm{~cm}^{-3}$ (Fig. 6). This agrees with the parameters derived by Henkel et al. (2008, 2009) and Muller et al. $(2011,2013)$ from multiline studies. 


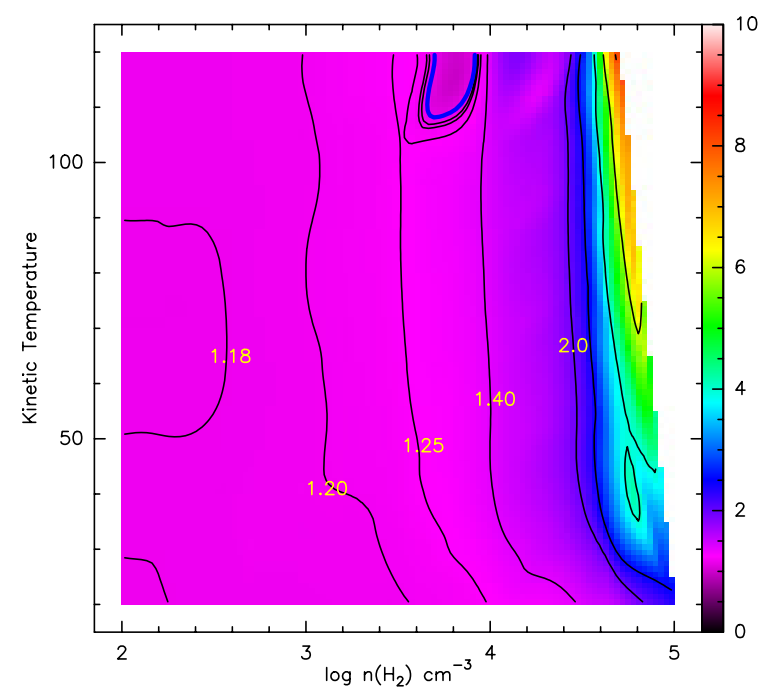

Fig. 6. $2_{02} \leftarrow 1_{11}$ to $1_{11} \leftarrow 0_{00}$ opacity ratios of cyclic paracyclopropenylidene (para $\mathrm{c}-\mathrm{C}_{3} \mathrm{H}_{2}$ ) under optically thin conditions as a function of density and temperature. Depending on the continnum source-covering factors used (see subsection $\mathrm{c}-\mathrm{C}_{3} \mathrm{H}_{2}$ in Sect. 4.2), we obtain ratios of $0.87 \pm 0.19$ and $1.14 \pm 0.25$. Both values are below the modeled ones, but accounting for the errors, they are not inconsistent with the standard values of $T_{\text {kin }}=50-80 \mathrm{~K}$ and $n\left(\mathrm{H}_{2}\right)=$ 1000-2000 cm $\mathrm{cm}^{-3}$ (Henkel et al. 2008, 2009; Muller et al. 2011, 2013).

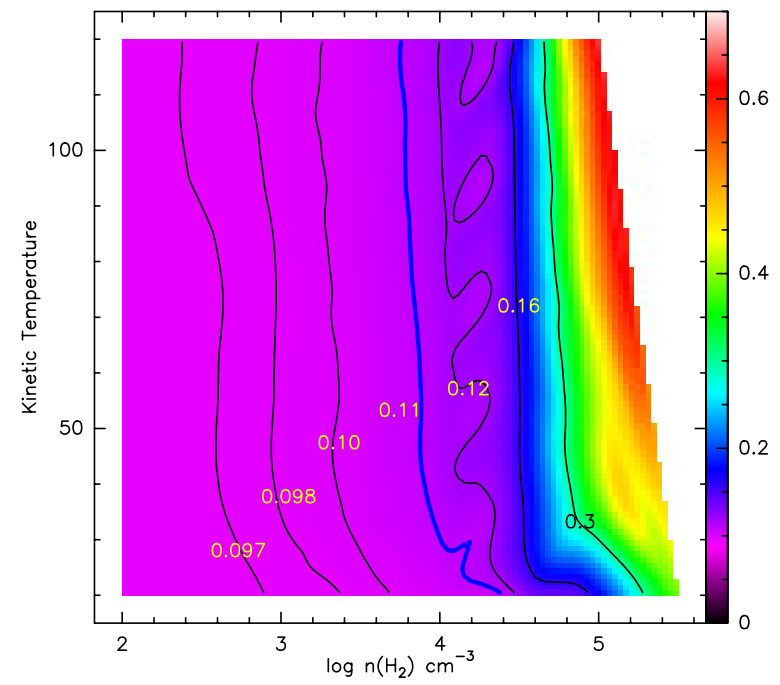

Fig. 7. $3_{12} \leftarrow 3_{03}$ to $2_{12} \leftarrow 1_{01}$ opacity ratios of cyclic orthocyclopropenylidene (ortho $\mathrm{c}-\mathrm{C}_{3} \mathrm{H}_{2}$ ) under optically thin conditions as a function of density and kinetic temperature. The blue almost vertical line represents the measured value $(0.110 \pm 0.015$; Sect. 4.2). When accounting for the error, this is consistent with the standard values given in the caption to Fig. 6.

(o): the measured ratio of the ortho $3_{12} \leftarrow 3_{03}$ to $2_{12} \leftarrow$ $1_{01}$ opacities, $0.110 \pm 0.015$ (both lines taken from Muller et al. $2011)$, is reproduced well with the same $T_{\text {kin }}$ and $n\left(\mathrm{H}_{2}\right)$ (Fig. 7) yielding a column density a few times that of the para type of the molecule (the precise factor depends on the $T_{\text {kin }}$ and $n\left(\mathrm{H}_{2}\right)$ parameters chosen), in accordance with the expected factor of three for the ortho/para abundance ratio.

Lines of this molecule commonly originate in moderately dense molecular gas (e.g., Thaddeus et al. 1995; Aladro et al. 2011), and our adopted temperature and density are in accordance with this scenario.

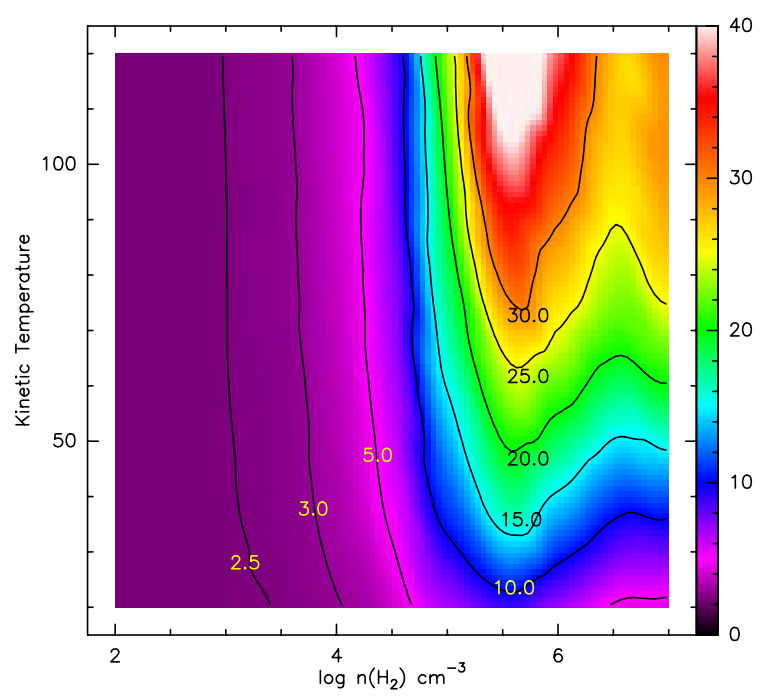

Fig. 8. $1_{0} \leftarrow 2_{-1}$ to $1_{0} \leftarrow 0_{0}$ opacity ratios of E-type methanol $\left(\mathrm{CH}_{3} \mathrm{OH}\right)$ under optically thin conditions as a function of density and kinetic temperature. The value of $1.8 \pm 0.4$ (Sect. 4.2), deduced from measurements, indicates a low density that is, within the error, consistent with the standard parameters given in Fig. 6.

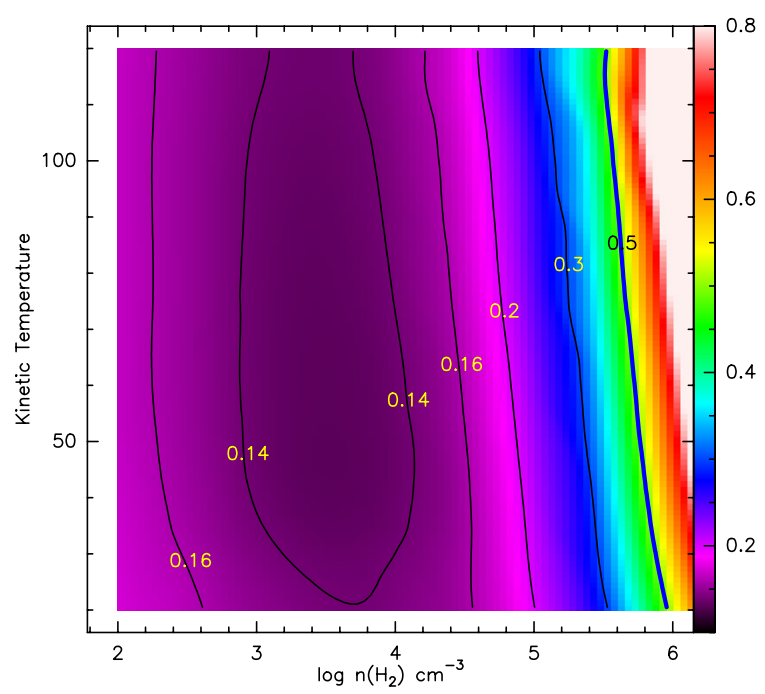

Fig. 9. $3_{12} \leftarrow 3_{13}$ to $2_{11} \leftarrow 2_{12}$ opacity ratios of ortho-formaldehyde (o- $\mathrm{H}_{2} \mathrm{CO}$ ) under optically thin conditions as a function of density and kinetic temperature. The blue thick almost vertical line on the right hand side denotes a line ratio of 0.5 (Sect. 4.2; the ratio obtained with frequency dependent continuum source-covering factors is $0.54 \pm 0.17$ ). Here the density is not consistent with the standard value given in the caption to Fig. 6.

Although the detection of the (Galactic) $\mathrm{C}_{3} \mathrm{H}_{2}\left(1_{10} \leftarrow 1_{01}\right)$ feature is tentative, it should be expected since this molecular transition is known to be quite widespread (e.g., Madden et al. 1989) and our source is located not far from the Galactic plane and center $\left(l^{\mathrm{II}}=12.2^{\circ}, b^{\mathrm{II}}=-5.7^{\circ}\right)$. We have checked other possible line identifications in the (Galactic) range of 18.335 to $18.353 \mathrm{GHz}$ as well as in the range 34.581 to $34.600 \mathrm{GHz}$, redshifted by $z=0.88582$, and find no other likely line candidate. There is no previous detection of Galactic molecular absorption lines along this line of sight.

$\mathrm{CH}_{3} \mathrm{OH}$ : quasi-thermal lines of methanol trace warm and at least moderately dense gas $\left(n\left(\mathrm{H}_{2}\right) \gtrsim 1000 \mathrm{~cm}^{-3}\right.$; e.g., Leurini et al. 2004). The species is easily dissociated, and it is not 


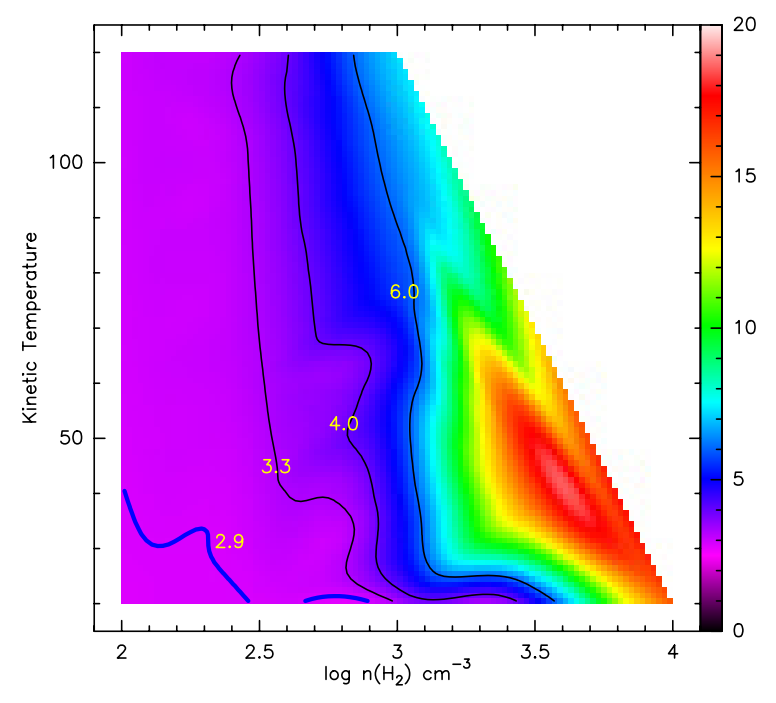

Fig. 10. $2_{1} \leftarrow 1_{0}$ to $1_{0} \leftarrow 0_{1}$ opacity ratios of sulfur monoxide (SO) under optically thin conditions as a function of density and kinetic temperature. With ratios of $2.0 \pm 0.5$ or $2.9 \pm 0.6$, depending on the adopted continuum cource covering factors, the density is below that of the standard value given in Fig. 6 . The thick blue line in the lower left corner denotes a ratio of 2.9 .

detected in Galactic clouds of very low density (Liszt et al. 2008). Applying the same kinetic temperature and density as above, the modeled ratio of the opacities of the molecular A and $\mathrm{E}$ species becomes 3.8 in case of equal abundances. This agrees within the errors with the observations (Table 2 and Fig. 1) and the more sensitive data from Bagdonaite et al. (2013a,b), taken more than a decade later. Referring to our clearly detected line of the A species (Fig. 1), dividing its intensity by 3.8 , and comparing it with that of the $\mathrm{E}\left(1_{0} \leftarrow 2_{-1}\right)$ transition observed by Muller et al. (2011) yields an opacity ratio of $1.8 \pm 0.4$, which is also consistent with the density estimate by Henkel et al. (2009; see Fig. 8). The kinetic temperature remains poorly constrained and allows for both $T_{\text {kin }}=50$ and $80 \mathrm{~K}$.

$\mathrm{H}_{2} \mathrm{CO}$ : formaldehyde can be efficiently produced on the surface of icy grains through hydrogenation of $\mathrm{CO}$ (e.g., Aladro et al. 2011). Interpolating the continuum flux density of the background source of PKS 1830-211 at 7.7 GHz from data at $5 \mathrm{GHz}$ and $8.4 \mathrm{GHz}$ (Subrahmanyan et al. 1990; Nair et al. 1993; Xu et al. 1995; van Ommen et al. 1995; Mattox et al. 1997; Lovell et al. 1998) yields 7.1 Jy for the frequency of the $\left(2_{11} \leftarrow 2_{12}\right)$ line observed by Menten et al. (1999). Their absorption feature of $0.073 \mathrm{Jy}$ therefore absorbs at its peak $\sim 1 \%$ of the total continuum flux density of PKS 1830-211. From that, the derived $\tau$ becomes 0.026 for a fixed $f_{\mathrm{c}}=0.38$. The observed $\tau$ ratio of the $3_{12} \leftarrow 3_{13}$ to $2_{11} \leftarrow 2_{12}$ lines is then of order unity while the model calculation for the temperature and density used above yields a ratio of 0.14 (Fig. 9). To fit a ratio of 1 to 2 , any temperature between 10 and $150 \mathrm{~K}$ would require densities $n\left(\mathrm{H}_{2}\right) \gtrsim 5 \times 10^{5} \mathrm{~cm}^{-3}$. However, accounting for the fact that the $2_{11} \leftarrow 2_{12}$ line is measured at low frequency $\left(v_{\text {observed }} \sim 7.68 \mathrm{GHz}\right)$ and that therefore the source-covering factor may be smaller, we obtain with the frequency dependent source-covering factor (see Sect. 4.1), originally proposed by Henkel et al. (2009), $f_{\mathrm{c}}=0.108$ and an optical depth of 0.10 . The resulting $3_{12} \leftarrow 3_{13}$ to $2_{11} \leftarrow 2_{12}$ ratio of optical depths would then become $\sim 0.54 \pm 0.17$, still favoring a density on the order of $10^{5} \mathrm{~cm}^{-3}$ (see Fig. 9), which would be consistent with the average density deduced by Mangum et al. (2013) from the $1_{10} \leftarrow 1_{11}$ and $2_{11} \leftarrow 2_{12}$ transitions of $\mathrm{H}_{2} \mathrm{CO}$ toward nearby actively star forming galaxies. If we adopt a dip half as deep for our line, the ratio would become $\sim 0.25-0.3$ and would reduce the density by half an order of magnitude, which is still rather large. To bring our so far preferred model (with $T_{\text {kin }}=80 \mathrm{~K}$ and $n\left(\mathrm{H}_{2}\right)=2000 \mathrm{~cm}^{-3}$ ) into rough accordance with the observations, our $3_{12} \leftarrow 3_{13}$ line should be weaker by a factor of $\sim 4$, which is inconsistent with the data (see Sect. 5 for a discussion of this result, which indicates strong deviations from the "canonical" physical parameters obtained in previous studies).

$S O$ : sulfur monoxide molecules are formed in a high temperature environment by neutral-neutral reactions with high activation energy (e.g., Takano et al. 1995). Both lines used for modeling (see Table 2) are from our observations. The line shapes appear to differ from those of the other transitions displayed in Fig. 1 showing a redshifted line wing. In case of the $2_{1} \leftarrow 1_{0}$ line, a small part of the redshifted absorption might be due to the $3_{03} \leftarrow 2_{02}$ line of cyanic acid (HOCN), which is displaced by $28.0 \mathrm{~km} \mathrm{~s}^{-1}$ and contains hyperfine structure components scattered over $10.7 \mathrm{~km} \mathrm{~s}^{-1}$. However, this molecule is rarely detected in the ISM (see Brünkens et al. 2010) and the profile of the other SO transition (the $1_{0} \rightarrow 0_{1}$ line) looks similar. Following Table 2, our measured $2_{1} \leftarrow 1_{0} / 1_{0} \leftarrow 0_{1}$ peak opacity ratios are $2.0 \pm 0.5\left(f_{\mathrm{c}}=0.227\right.$ and 0.156$)$ and $2.9 \pm 0.6$ $\left(f_{\mathrm{c}}=0.38\right)$. With $T_{\text {kin }}=80 \mathrm{~K}$, gas densities become very low (a few $100 \mathrm{~cm}^{-3}$ or less) to fit the observed line ratio (see Fig. 10), which is much lower than the one suggested by, for example, Henkel et al. (2009). On the other hand, choosing a value near their $n\left(\mathrm{H}_{2}\right)=2000 \mathrm{~cm}^{-3}$, the modeled gas temperature drops to $\$ 20 \mathrm{~K}$, in strong contradiction to $\mathrm{NH}_{3}$ and $\mathrm{CH}_{3} \mathrm{CN}$ data (Henkel et al. 2008; Muller et al. 2011).

The two modeled cases with different source-covering factors are in accordance with the upper limit of $\tau$ determined from the nondetection of the $4_{3} \leftarrow 3_{3}$ line. The only possible assumption to reconcile the result related to the narrow component with those from $\mathrm{NH}_{3}, \mathrm{HC}_{3} \mathrm{~N}$, and $\mathrm{CH}_{3} \mathrm{CN}$ (Henkel et al. 2008, 2009; Muller et al. 2011, 2013), and our results from $\mathrm{C}_{3} \mathrm{H}_{2}$ and $\mathrm{CH}_{3} \mathrm{OH}$ is that the absorbing molecular gas shows strong gradients at least in density and possibly also in $T_{\text {kin }}$ (see also Sect. 5).

\subsection{Column densities and abundances}

Our modeled molecular column densities are presented in Table 4 along with abundance ratios relative to $\mathrm{CO}$ and $\mathrm{H}_{2}$. Our abundance ratios are generated using $N_{\mathrm{CO}}=3 \times 10^{18} \mathrm{~cm}^{-2}$ (Gerin et al. 1997) and $N_{\mathrm{H}_{2}}=3 \times 10^{22} \mathrm{~cm}^{-2}$ (Gerin et al. 1997; Wiklind \& Combes 1996a). This is also discussed by Muller et al. (2011). Depending on the background source-covering factor $\left(f_{\mathrm{c}}=0.38\right.$ or frequency dependent; see Table 2), there is in most cases an uncertainty by a factor of almost two in the column densities. Here we refer to average values between these two extremes, accordingly providing $\pm 40 \%$ errors.

To compare our results with those of Menten et al. (1999) obtained at the VLA, we take molecular column densities and their ratios to $\mathrm{CO}$ directly from their Table 1, while we derive their ratio to $\mathrm{H}_{2}$ using the $\mathrm{H}_{2}$ column density mentioned above. For comparison with results by Muller et al. (2011), we use their $N_{\text {mol }}$ from their Table 3 , and $N_{\text {CO }}$ (as mentioned above) from Wiklind \& Combes (1996a) and Gerin et al. (1997). Our chosen $N_{\mathrm{H}_{2}}$ value is $50 \%$ higher than than the value adopted by Muller et al. (2011) in their Table 8.

$c-\mathrm{C}_{3} \mathrm{H}_{2}$ : our total column density is about an order of magnitude higher than in Menten et al. (1999), but is in good 
Table 4. Kinetic temperatures, densities, column densities $N$, and fractional abundances $N / N_{\mathrm{CO}}$ and $N / N_{\mathrm{H}_{2}}$.

\begin{tabular}{|c|c|c|c|c|c|c|c|c|}
\hline Molecule & $\begin{array}{c}\text { Temperature } \\
(\mathrm{K})\end{array}$ & $\begin{array}{l}\text { Gas density } \\
\left(\mathrm{cm}^{-3}\right)\end{array}$ & $\begin{array}{l}N \\
\left(10^{12} \mathrm{c}\right.\end{array}$ & $\begin{array}{ll}\left.\mathrm{a}^{-2}\right) & N_{\text {Lit. }}\end{array}$ & $\begin{array}{r}N / N_{\mathrm{CO}} \\
\left(10^{-6}\right)\end{array}$ & $\begin{array}{c}N / N_{\mathrm{CO}}(\text { Lit. }) \\
\left(10^{-6}\right)\end{array}$ & $\begin{array}{l}N / N_{\mathrm{H}_{2}} \\
\left(10^{-9}\right)\end{array}$ & $\begin{array}{c}N / N_{\mathrm{H}_{2}} \text { (Lit.) } \\
\left(10^{-9}\right)\end{array}$ \\
\hline $\mathrm{o}+\mathrm{p} \mathrm{c}-\mathrm{C}_{3} \mathrm{H}_{2}$ & 80 & 2000 & $65( \pm 40 \%)$ & $\begin{array}{r}7(\mathrm{Me}) \\
53(\mathrm{Mu})\end{array}$ & 22 & $\begin{array}{r}2(\mathrm{Me}) \\
17(\mathrm{Mu})\end{array}$ & 2.2 & $\begin{array}{r}0.23(\mathrm{Me}) \\
1.8(\mathrm{Mu})\end{array}$ \\
\hline $\mathrm{CH}_{3} \mathrm{OH}$ & 80 & 2000 & $140( \pm 40 \%)$ & $170(\mathrm{Mu})$ & 45 & $57(\mathrm{Mu})$ & 4.5 & $5.6(\mathrm{Mu})$ \\
\hline $\mathrm{o}-\mathrm{H}_{2} \mathrm{CO}$ & $10-150$ & $>5 \times 10^{4}$ & $400( \pm 40 \%)$ & $\begin{array}{r}380(\mathrm{Me}) \\
60(\mathrm{Mu})\end{array}$ & 130 & $\begin{array}{r}130(\mathrm{Me}) \\
20(\mathrm{Mu})\end{array}$ & 13.0 & $\begin{array}{r}13(\mathrm{Me}) \\
2(\mathrm{Mu})\end{array}$ \\
\hline $\mathrm{SO}$ & $20-80$ & $400-2000$ & $35( \pm 50 \%)$ & $26(\mathrm{Mu})$ & 12 & $9(\mathrm{Mu})$ & 1.2 & $0.9(\mathrm{Mu})$ \\
\hline SiS & 80 & 2000 & $<5$ & & $<2.5$ & & $<0.2$ & \\
\hline $\mathrm{HNCO}$ & 80 & 2000 & $<8.6$ & $6.2(\mathrm{Mu})$ & $<4$ & $2.1(\mathrm{Mu})$ & $<0.3$ & $0.2(\mathrm{Mu})$ \\
\hline
\end{tabular}

Notes. Column (1): molecular species; Col. (2): adopted kinetic temperature; Col. (3): adopted gas density; Col. (4): our column densities; Col. (5): column densities derived from data of Menten et al. (1999) (Me) and Muller et al. (2011) (Mu) in the way described in Sect. 4.3; Cols. (6) and (7): same as Cols. (4) and (5), but relative to the adopted CO column density, $N_{\mathrm{CO}}=3 \times 10^{18} \mathrm{~cm}^{-3}$ (Sect. 4.3); Cols. (8) and (9): same as Cols. (4) and (5), but relative to the adopted $\mathrm{H}_{2}$ column density, $N_{\mathrm{H}_{2}}=3 \times 10^{22} \mathrm{~cm}^{-3}$ (see Sect. 4.3).

agreement with that of Muller et al. (2011). These factors also apply to the ratios relative to $\mathrm{CO}$ and $\mathrm{H}_{2}$.

$\mathrm{CH}_{3} \mathrm{OH}$ : our column density and relative abundance are in close accordance with Muller et al. (2011) and also with Bagdonaite et al. (2013a,b).

$\mathrm{H}_{2} \mathrm{CO}$ : our values agree well with those of Menten et al. (1999), while those of Muller et al. (2011) are almost an order of magnitude lower.

$S O$ : our column density is in accordance with the value given by Muller et al. (2011). Their abundance relative to $\mathrm{H}_{2}$ is almost identical to our result. For all temperatures between $20 \mathrm{~K}$ and $80 \mathrm{~K}$, resulting column densities are similar within a factor of $<2$.

SiS, HNCO: our nondetections yield upper limits to the molecule's abundances relative to $\mathrm{H}_{2}$. The one for $\mathrm{HNCO}$ is in accordance with the value derived by Muller et al. (2011) from a detected $J=3 \leftarrow 2$ line.

\subsection{Excitation temperatures}

Some of the molecular lines we have measured exhibit excitation temperatures, which are quite far from the expected $T_{\mathrm{CMB}}$ value, $T_{\mathrm{CMB}}=2.73 \mathrm{~K} \times(1+z)=5.14 \mathrm{~K}$ (Muller et al. 2013; Sato et al. 2013). The $J_{\mathrm{KaKc}}=3_{12} \leftarrow 3_{13}$ line of $\mathrm{H}_{2} \mathrm{CO}$, for example, is collisionally cooled (e.g., Evans et al. 1975; Zeiger \& Darling 2010; Darling \& Zeiger 2012) and may show excitation temperatures well below $5.14 \mathrm{~K}$ in spite of the uncertainties with respect to density (Sect. 4.2). The second example is the SO $1_{0} \leftarrow 0_{1}$ transition. After we apply our LVG code, it exhibits excitation temperatures of several $10 \mathrm{~K}$ or even inversion in case of $T_{\mathrm{CMB}}<4.0 \mathrm{~K}$ for the standard parameters $T_{\mathrm{kin}}=80 \mathrm{~K}$ and $n\left(\mathrm{H}_{2}\right)=2000 \mathrm{~cm}^{-3}$. In the case of lower $T_{\text {kin }}$ and density, however, for $40 \mathrm{~K}$ and $1000 \mathrm{~cm}^{-3}$ (see Sect. 4.2), for example, the excitation temperature becomes "well behaved" for $3 \mathrm{~K}<T_{\mathrm{CMB}}<$ $7 \mathrm{~K}$, only surpassing the $T_{\mathrm{CMB}}$ value by $2.0-2.5 \mathrm{~K}$. On the other hand, the LVG calculations indicate that the excitation temperatures of para and ortho $\mathrm{c}-\mathrm{C}_{3} \mathrm{H}_{2}, \mathrm{CH}_{3} \mathrm{OH}$, and the $2_{1} \leftarrow 1_{0}$ transition of $\mathrm{SO}$ are normal in the sense that they are slightly higher than the $T_{\mathrm{CMB}}$ value and rise with increasing density (cf. Fig. 3 of Muller et al. 2013). For $n\left(\mathrm{H}_{2}\right)=2000 \mathrm{~cm}^{-3}, T_{\text {kin }}=80 \mathrm{~K}$, and
$3 \mathrm{~K}<T_{\mathrm{CMB}}<7 \mathrm{~K}$, the $\mathrm{C}_{3} \mathrm{H}_{2}$ and $1_{0} \leftarrow 0_{0} \mathrm{CH}_{3} \mathrm{OH}$ (E) excitation temperatures are within $5 \%$ and slightly above the chosen cosmic microwave value. This emphasizes that these lines are suitable tools for constraining $T_{\mathrm{CMB}}$ at redshift $z \sim 0.89$ and confirms the assumption of $T_{\text {ex }} \ll T_{\mathrm{C}}\left(T_{\mathrm{C}} \gtrsim 10^{7} \mathrm{~K}\right.$; e.g., Jin et al. 2003), used to calculate optical depths (see Sect. 3).

\section{Discussion}

The frequencies of our observations range from 15 to $35 \mathrm{GHz}$, those of Muller et al. (2011) and Menten et al. (1999) from 30 to $50 \mathrm{GHz}$ with the exception of the $\mathrm{H}_{2} \mathrm{CO}\left(2_{11} \leftarrow 2_{12}\right)$ line at 7.7 GHz. The size of the SW spot of the background continuum source lensed by the foreground galaxy is reported to decrease with frequency (e.g. Carilli et al. 1998; Garrett et al. 1998; Jin et al. 2003); but the sizes given there for a specific frequency do not agree with each other. Nevertheless, at the highest observing frequency of $45 \mathrm{GHz}$ discussed in our analysis, this size must be larger than 0.2 milliarcsec, which corresponds to $\sim 2$ pc linear extent.

Within the limitations by noise, all our spectra as well as the used spectra of the referenced literature show compatible center velocities (see, e.g., also Henkel et al. 2008; and Muller et al. 2014b). Therefore, we assume that they all originate in the same cloud complex. In particular, we also find that the line widths do not depend on the frequency of the studied feature (see also Henkel et al. 2008, 2009 for complementary lines). Observing molecular cloud material of $\gtrsim 2 \mathrm{pc}$ linear extent along a line of sight with a likely larger depth, a line width of $\sim 10$ $20 \mathrm{~km} \mathrm{~s}^{-1}$ (neglecting the $V \sim 175 \mathrm{~km} \mathrm{~s}^{-1}$ component reported by Muller et al. 2014b) consisting of several kinematic subcomponents (e.g., Muller et al. 2008; Murphy et al. 2008; Henkel et al. 2009) could be readily expected by a combination of "normal" clouds (e.g., Larson 1981).

From their ammonia multiline analysis, Henkel et al. (2008) conclude that $80 \%$ to $90 \%$ of the ammonia gas has a kinetic temperature of about $80 \mathrm{~K}$. But since rotational temperatures of the ammonia gas vary from $35 \mathrm{~K}$ to up to $600 \mathrm{~K}$, they also state that the gas may show large temperature gradients. Our line analysis above for the first time indicates strong density 
gradients (for variations by a factor of two, see also Muller et al. 2013). As Henkel et al. (2008) point out, kinetic temperatures are far too high for a dark-cloud-scenario. The different temperature/density parameters obtained instead indicate a combination of outer cloud layers and/or intercloud regions as well as denser cloud cores.

This "mixture" of physical properties is also supported by a comparison of molecular abundances with those of different types of Galactic objects. For the following comparison of our abundance values to those of sources with different characters, we use values of different cloud types contained in Table 8 of Muller et al. (2011) for our observed molecules. There is an overlap with Table 1 of Menten et al. (1999) only for the source TMC 1 and for the molecules $\mathrm{C}_{3} \mathrm{H}_{2}$ and $\mathrm{H}_{2} \mathrm{CO}$; these values agree with each other.

Our $\mathrm{C}_{3} \mathrm{H}_{2}$ abundance agrees with those of low density molecular clouds and lies between those of the starburst galaxies NGC 253 and NGC 4945. Fractional abundances for both translucent clouds and TMC 1 are much higher. For $\mathrm{CH}_{3} \mathrm{OH}$, our abundance is similar to that of NGC 4945, in between those of translucent clouds and TMC 1, whereas that of NGC 253 is a moderate factor of 2 higher. The $\mathrm{H}_{2} \mathrm{CO}$ abundance is an order of magnitude higher than in the starburst galaxies, half an order of magnitude higher than in diffuse and translucent clouds, and is within the uncertainties (factors of two) compatible with that of TMC 1. Our SO abundance agrees with those of both starburst galaxies, whereas those of translucent clouds and the TMC 1 are higher. In contrast, our upper limit as well as the value for HNCO (Muller et al. 2011) agrees with the values of the TMC 1 and Sgr B2; here, abundances of translucent clouds and the starburst galaxies are higher.

All this increasingly indicates that former interpretations attempting to attribute observed molecular abundances to one distinct cloud type do not lead to a realistic scenario of the ISM we observe toward the southwestern molecular hotspot of PKS 1830-211. We possibly observe a cloud complex that is, with respect to spatial fine structure, similar to cloud complexes in our own Galaxy. With the dust-to-gas ratio in PKS 1830-211 being consistent with the Galactic value (Dai et al. 2006; Aller et al. 2012), we apparently observe a combination of different conditions in different cloud types that should not be surprising for a region that encompasses several pc in the plane of the sky and presumably an even larger linear scale along the line of sight.

Not yet aware of the $V \sim 175 \mathrm{~km} \mathrm{~s}^{-1}$ feature reported by Muller et al. (2014b), Henkel et al. (2008) discuss the scenario that the southwestern absorbing cloud toward PKS 1830-211 may be similar to the gas seen toward Sgr B2 in our Galaxy. What they miss is a similar molecular condensation located at several kpc from the center as seen toward PKS 1830-211. Line widths of various species observed on various linear scales in Sgr B2 (0.4-2.5 pc) are approximately $20 \mathrm{~km} \mathrm{~s}^{-1}$ (Rohlfs et al. 2010; Comito et al. 2003). Apparently at $\sim 7$ Gyr in the past, a cloud complex at 2-3 kpc galactocentric distance can also show this fingerprint.

\section{Conclusions}

From our excitation analysis of molecular line spectra of various species applying a large velocity gradient radiative transfer model, we draw the following conclusions:

- For the $\mathrm{c}-\mathrm{C}_{3} \mathrm{H}_{2}$ and $\mathrm{CH}_{3} \mathrm{OH}$ molecules, the data are compatible with $T_{\text {kin }}=80 \mathrm{~K}$ and $n\left(\mathrm{H}_{2}\right)=2000 \mathrm{~cm}^{-3}$, in accordance with previously reported results, indicating gas of low density.

- While our observed $\mathrm{H}_{2} \mathrm{CO} \mathrm{K}$-doublet line is noisier than that of Menten et al. (1999), the resulting $\tau$ values of both lines are very similar and yield volume densities $n\left(\mathrm{H}_{2}\right) \gtrsim$ $5 \times 10^{5} \mathrm{~cm}^{-3}$ for all kinetic temperatures between $10 \mathrm{~K}$ and $80 \mathrm{~K}$. An absorption dip half as deep as was derived from our data, which still might be adopted as an extreme, would reduce the density to $n\left(\mathrm{H}_{2}\right) \sim 5 \times 10^{4} \mathrm{~cm}^{-3}$, which still is more than an order of magnitude higher than the one derived by Henkel et al. (2009) on the basis of an $\mathrm{HC}_{3} \mathrm{~N}$ multiline analysis.

- For the SO molecule, if we assume $T_{\text {kin }}=80 \mathrm{~K}$, the volume gas density becomes extremely low $\left(n\left(\mathrm{H}_{2}\right) \lesssim 500 \mathrm{~cm}^{-3}\right)$. If we raise the density to $2000 \mathrm{~cm}^{-3}$, the kinetic temperature drops to $20 \mathrm{~K}$. Both models, as well as those between these two extremes, result in about the same column density (see Table 4).

- Our resulting densities and relative molecular abundances are in fair if not good agreement with those found in the local universe. This accords with the statement of Aller et al. (2012) that "there is also evidence that the dust-to-gas ratio in this system is consistent with the Galactic value".

- For the southwestern hotspot of PKS 1380-211, there is a trend toward rising integrated opacities during the late nineties, with peaks near 2001, followed by a minimum around 2006. In recent years, opacities were rising again, reaching a maximum in 2011/2012. In the long term we may test for periodicities, possibly probing a putative precessing jet in the background blazar. In this context, long term monitoring of both the southwestern and northeastern velocity components would be instructive.

- Molecules are known to have different abundances in different types of clouds (e.g., Menten et al. 1999; Muller et al. 2011). Together with the fact that the lines analyzed here reflect different physical conditions, we conclude that, within the observed region of a few pc in size along the plane of the sky and likely a larger depth along the line of sight, there are strong gradients in density and likely also in temperature. To summarize, we see a combination of conditions typical of different types of molecular gas.

Acknowledgements. We wish to thank an anonymous referee for useful comments. We used NASA's Astrophysical Data System (ADS), the Cologne Database for Molecular Spectroscopy (CDMS; see Müller et al. 2001, 2005), the JPL Catalog (http://spec.jpl.nasa.gov/ftp/pub/catalog/catform. $\mathrm{html}$ ), and the line lists of Lovas (1992) and Coudert \& Roueff (2006).

\section{References}

Aladro, R., Martin-Pintado, J., Martin, S., Mauersberger, R., \& Bayet, E. 2011, A\&A, 525, A89

Aller, M. C., Kulkarni, V. P., York, D. G., et al. 2012, ApJ, 748, 19

Bagdonaite, J., Jansen, P., Henkel, C., et al. 2013a, Science, 339, 46

Bagdonaite, J., Daprà, M., Jansen, P. et al. 2013b, Phys. Rev. Lett., 111, 231101

Brünkens, S., Belloche, A., Martín, S., Verheyen, L., \& Menten, K. M. 2010, A\&A, 516, A109

Carilli, C. L., Menten, K. M., Reid, M. J., Rupen, M., \& Claussen, M. 1998, in Radio Emission from Galactic and Extragalactic Compact Sources, eds. A. Zensus et al., ASP Conf. Ser., 144, 317

Comito, C., Schilke, P., Gerin, M., et al. 2003, A\&A 402, 635

Coudert, L. H., \& Roueff, E. 2006, A\&A, 449, 855

Courbin, F., Meylan, G., Kneib, J.-P., \& Lidman, C. 2002, ApJ, 575, 95

Dai, X., Chartas, C. S., \& Mathur, S. 2006, ApJ, 637, 53

Darling, J., Zeiger, B. 2012, ApJ, 740, L33

De Rosa, A., Piro, L., Tramacere, A., et al. 2005, A\&A, 438, 121

Evans, N. J., Morris, G., Sato, T, \& Zuckerman, B. 1975, ApJ, 196, 433 
Garrett, M. A., Leppänen, K., Porcas, R. W, et al. 1998, in Radio Emission from Galactic and Extragalactic Compact Sources, eds. A. Zensus et al., ASP Conf. Ser., 144,313

Gerin, M., Phillips, T. G., Benford, D. J., et al. 1997, ApJ, 488, L31

Henkel, C., Braatz, J. A., Menten, K. M., \& Ott, J. 2008, A\&A, 485, 451

Henkel, C., Menten, K. M., Murphy, M. T., et al. 2009, A\&A, 500, 725

Jauncey, D. L., Reynolds, J. E., Tzioumis, A. K., et al. 1991, Nature, 352, 132

Jin, C., Garrett, M. A., Nair, S., et al. 2003, MNRAS, 340, 1309

Kanekar, N., Carilli, C. L., Langston, G. I., et al. 2005, Phys. Rev. Lett., 95, 261301

Leurini, S., Schilke, P., Menten, K. M., et al. 2004, A\&A, 422.573

Lidman, C., Courbin, F., Meylan, G., et al. 1999, ApJ, 514, L57

Liszt, H. S., Pety, J., \& Lucas, R. 2008, A\&A, 486, 493

Lovell, J. E. J., Jauncey, D. L., \& Reynolds, J. E., 1998, ApJ, 508, L51

Larson, R. B. 1981, MNRAS, 194, 809

Lovas, F. J. 1992, J. Phys. Chem. Ref. Data, 21, 181

Madden, S. C., Irvine, W. M., Matthews, H. E., Friberg, P., \& Swade, D. A. 1989, AJ, 97, 1403

Mangum, J., Darling, J., Henkel, C., \& Menten, K. M. 2013, ApJ, 766, 108

Martí-Vidal, I., Muller, S., Combes, F., et al. 2013, A\&A, 558, A123

Mattox, J. R., Schachter, J., Molnar, L., Hartman, R. C., \& Patnaik, A. R. 1997, ApJ, 481, 95

Menten, K. M., Carilli, C., \& Reid, M. J. 1999, in Highly Redshifted Radio Lines, eds. C. Carilli, et al., ASP Conf. Ser., 156, 218

Menten, K. M., Güsten, R., Leurini, S., et al. 2008, A\&A, 492, 725

Müller, H. S. P, Thorwirth, S., Roth, D. A., \& Winnewisser, G. 2001, A\&A, 370, L49

Müller, H. S. P, Schlöder, F., Stutzki, J., \& Winnewisser, G. 2005, J. Mol. Struct. 742,215
Muller, S., \& Guélin, M. 2008, A\&A, 491, 739

Muller, S., Guélin, M., Dumke, M., Lucas, R., \& Combes, F. 2006, A\&A, 458, 417

Muller, S., Beelen, A., Guélin, M., et al. 2011, A\&A, 535, A103

Muller, S., Beelen, A., Black, J. H., et al. 2013, A\&A, 551, A109

Muller, S., Black, J. H., Guélin, M., et al. 2014a, A\&A, 566, L6

Muller, S., Combes, F., Guélin, M., et al. 2014b, A\&A, 566, A112

Murphy, M. T., Flambaum, V. V., Muller, S., \& Henkel, C. 2008, Science, 320, 1611

Nair, S., Narasimha, D., \& Rao, A. P. 1993, ApJ, 407, 46

Nair, S., Jin, C., \& Garrett, M. A. 2005, MNRAS, 362, 1157

Planck Collaboration XVI. 2014, A\&A, 571, A16

Rolffs, R., Schilke, P., Comito, C., et al. 2010, A\&A, 521, L46

Sato, M., Reid, M. J., Menten, K. M., \& Carilli, C. L. 2013, ApJ, 764, 132

Subrahmanyan, R., Narashima, D., Rao, A. P., \& Swarup, G. 1990, MNRAS, 246,263

Takano, S., Nakai, N., \& Kawaguchi, K. 1995, PASJ, 47, 801

Thaddeus, P., Vrtilek, \& J. M., Gottlieb, C. A. 1985, ApJ, 299, L63

van Ommen, T. D., Jones, D. L., Preston, R. A., \& Jauncey, D. L. 1995, ApJ, 444,561

Wiklind, T., \& Combes, F. 1994, A\&A, 286, L9

Wiklind, T., \& Combes, F. 1995, A\&A, 299, 382

Wiklind, T., \& Combes, F. 1996a, Nature, 379, 139

Wiklind, T., \& Combes, F. 1996b, A\&A, 315, 86

Wiklind, T., \& Combes, F. 1998, ApJ, 500, 129

Winn, J. N., Lovell, J. E. J., Chen, H.-W., et al. 2002, ApJ, 564, 143

Xu, W., Readhead, A. C. S., Pearson, T. J., Polatidis, A. G., \& Wilkinson, P. N. 2005, ApJS, 99, 297

Zeiger, B., \& Darling, J. 2010, ApJ, 709, 386 